\title{
One-Pot Pyrrolidine-Catalyzed Synthesis of Benzopyrans, Benzothiopyranes, and Dihydroquinolidines
}

\author{
Ismail Ibrahem, Henrik Sundén, Ramon Rios, Gui-Ling Zhao, and Armando Córdova*
}

\begin{abstract}
A simple catalytic racemic synthesis of benzopyranes, benzothiopyranes and 1,2-dihydroquinolines is presented. The organocatalytic domino reactions between 2-heteroatom substituted aldehyde derivatives and $\alpha, \beta$-unsaturated aldehydes or $\alpha, \beta$-unsaturated cyclic ketones proceed with excellent chemoselectivity to give the corresponding products in good to high yields.
\end{abstract}

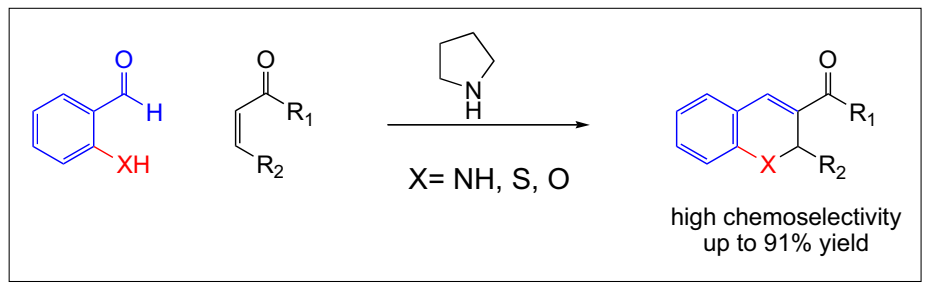

Keywords: Asymmetric synthesis · Organocatalytic domino reactions · Pyrrolidine

\section{Introduction}

Heterocycles are of immense importance in the design and discovery of new compounds for pharmaceutical applications. ${ }^{[1]}$ Benzopyranes, ${ }^{[2]}$ benzothiopyranes ${ }^{[3]}$ and dihydroquinolines ${ }^{[4]}$ are valuable compounds that have attracted much attention from a broad area of science, including medicinal chemistry, physical chemistry, synthetic organic chemistry and natural product chemistry due to their biological activities, distinctive structures and the potential for further transformations. Thus, several methods have been developed for the synthesis of this type of heterocycle. ${ }^{[5]}$ In this context, Bräse and co-workers described

\footnotetext{
${ }^{\star}$ Correspondence: Prof. Dr. A. Córdova Department of Organic Chemistry Arrhenius Laboratory

Stockholm University

SE-106 91 Stockholm

Sweden

Fax: +64 8154908

E-Mail: acordova1a@netscape.net
}

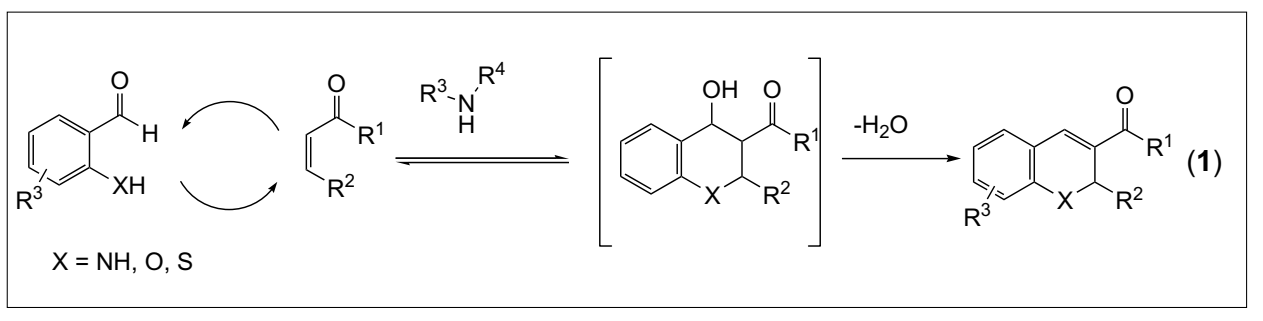

Scheme 1.

an elegant route to tetrahydroxanthenones and chromenes based on the 1,4-diazabicyclo[2.2.2] octane (DABCO) mediated reaction between salicylic aldehyde derivatives and $\alpha, \beta$-unsaturated ketones and aldehydes, respectively. This reaction proceeds via a Baylis-Hillman reaction pathway. ${ }^{[6]}$ More recently, Shi and co-workers reported a new approach to the synthesis of tetrahydroxanthenones using $\mathrm{N}$-tosylimines as substrates and dimethylphenylphosphine as the catalyst. ${ }^{[7]}$

Recently, organocatalytic asymmetric transformations that involve catalytic domino or cascade reactions via enamine and iminium intermediates were reported. ${ }^{[8,9]}$ However, to the best of our knowledge, there are no reports on the synthesis of racemic chromene, thiochromene, dihydroquinoline, tetrahydroxanthenone and tetra- hydrothioxanthenone derivatives based on this concept. ${ }^{[10]}$ Encouraged by the research on organocatalytic domino reactions, we envisioned a simple catalytic route to the synthesis of different heterocycles by organocatalytic domino reactions between 2-heteroatom substituted benzaldehydes 1 and enals $\mathbf{2}$ or cyclic enones $\mathbf{3}$ via iminium and enamine activation (Scheme 1).

Herein, we present the one-pot pyrrolidine-catalyzed synthesis of chromenes, thiochromenes, 1,2-dihydroquinolines, tetrahydroxanthenones and tetrahydrothioxanthenones in good to excellent yields.

\section{Results and Discussion}

In an initial screen, we found that pyrrolidine catalyzed the reaction between 
Table 1. Direct organocatalytic domino oxo-Michael/aldol condensation between hydroxybenzaldehydes 1 and $\alpha, \beta$-unsaturated aldehydes $\mathbf{2}^{\mathrm{a}}$

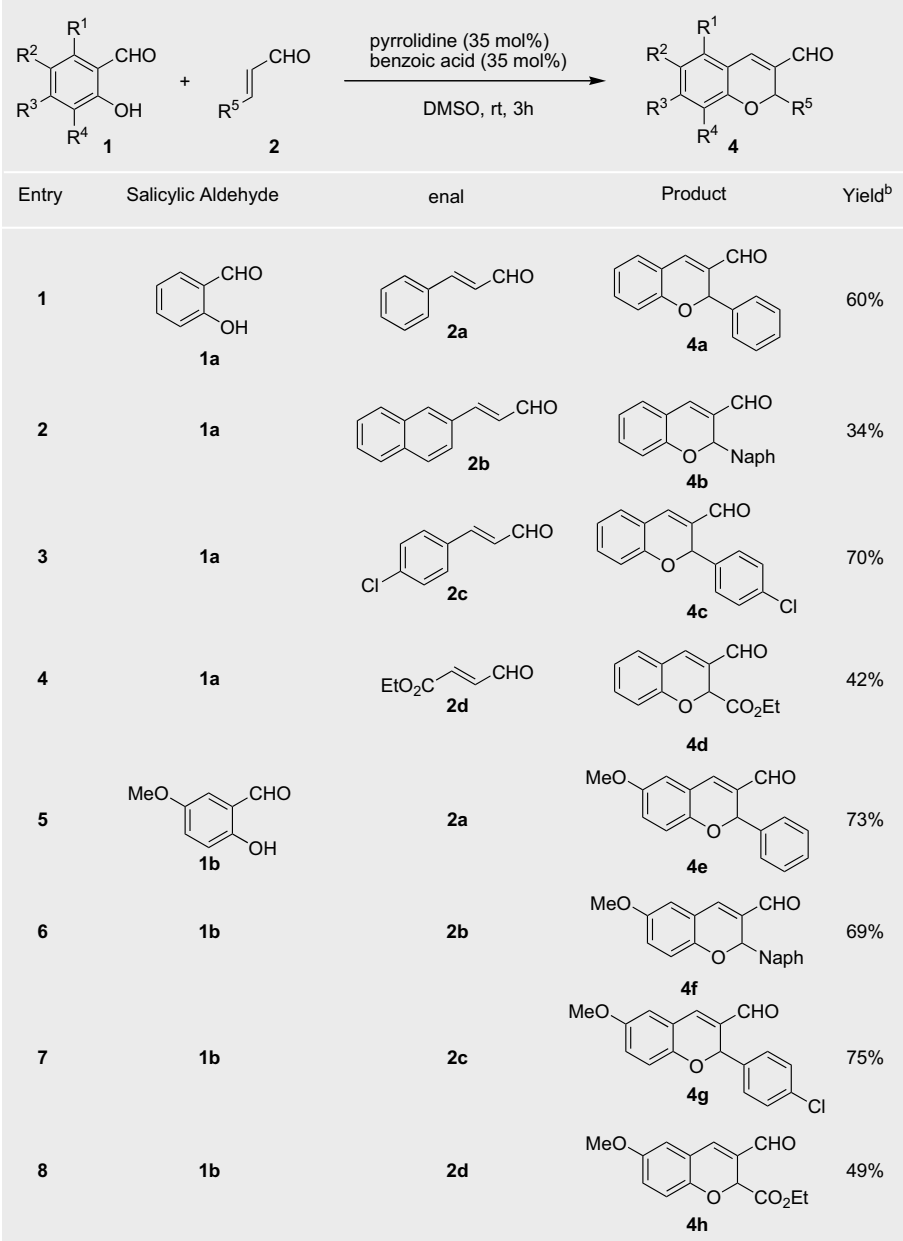

aExperimental conditions: A mixture of $1(0.3 \mathrm{mmol}), 2(0.25 \mathrm{mmol})$, benzoic acid (35 mol\%) and pyrrolidine (35 mol\%) in $1 \mathrm{ml}$ of DMSO was

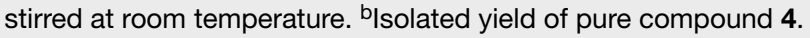

Table 2. Direct organocatalytic domino thia-Michael/aldol condensation between 2-mercaptobenzaldehyde $1 \mathrm{c}$ and $\alpha, \beta$-unsaturated aldehydes $\mathbf{2}^{\mathrm{a}}$

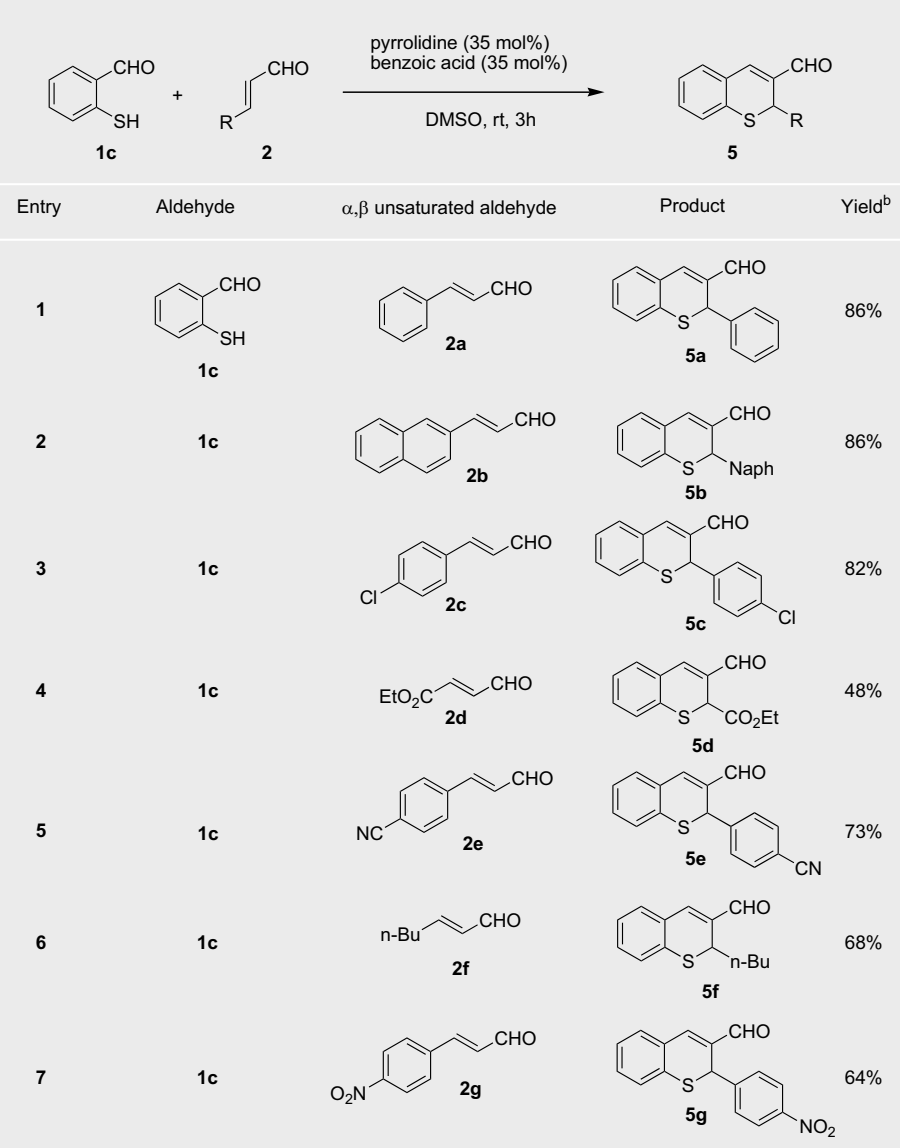

aExperimental conditions: A mixture of $1 \mathrm{c}(0.3 \mathrm{mmol}), 2(0.25 \mathrm{mmol})$, benzoic acid (35 mol\%) and pyrrolidine (35 mol\%) in $1 \mathrm{ml}$ of DMSO was stirred at room temperature. blsolated yield of pure compound 5 . 2-hydroxybenzaldehyde 1 a $(0.30 \mathrm{mmol})$ and cinnamic aldehyde $2 \mathbf{a}(0.25 \mathrm{mmol})$ with high chemoselectivity in different solvents to give the corresponding chromene $\mathbf{4 a}$ in good yields. To our delight, we found that the best results were obtained in DMSO with pyrrolidine as the catalyst (35 $\mathrm{mol} \%$ ) and benzoic acid (35 mol\%) as the additive at room temperature. Under these conditions, pyrrolidine catalyzed the formation of the corresponding chromene $4 \mathbf{a}$ in $60 \%$ yield. Encouraged by this result, we decided to investigate the catalytic domino oxoMichael/aldol reaction between 1a and different $\alpha, \beta$-unsaturated aldehydes (Table 1).

The reactions were highly chemoselective and gave the corresponding chromene3-carbaldehydes $\mathbf{4 a - 4 h}$ in moderate to high yields. We next investigated the domino reaction between 2-mercaptobenzaldehyde $1 \mathbf{c}$ and $\alpha, \beta$-unsaturated aldehydes 2 vide infra (Table 2).

The reactions were more efficient compared to the domino reactions with 2-hydroxybenzaldehyde and furnished the corresponding thiochromenes derivatives $\mathbf{5}$ in high yields. For instance thiochromene 5b was assembled in $86 \%$ yield.

Encouraged by the reports of the Hamada group, ${ }^{[5 v-w]}$ we next investigated the domino reaction between 2-aminobenzaldehydes derivatives and $\alpha, \beta$-unsaturated aldehydes (Table 3 ). This is a more challenging reaction, since competing imine formation could possibly occur (Scheme 2).

Happily, the reactions proceeded smoothly and the corresponding racemic 1,2-dihydroquinolines $\mathbf{6}$ were assembled in $56-79 \%$ yield. Thus, pyrrolidine was a highly chemoselective catalyst for this domino aza-Michael/aldol condensation reaction.

Next, we explored the scope of the pyrrolidine-catalyzed domino reaction using $\alpha, \beta$-unsaturated ketones as the substrates. We first investigated the reaction between 2-mercaptobenzaldehyde 1c and 2-cyclohexene-1-one 3a. After screening several reaction conditions, we found that the best results were obtained when pyrrolidine (20 mol\%) was used as the catalyst and benzoic acid $(20 \mathrm{~mol} \%)$ was used as an additive in<smiles>NC1=C(C=O)C=C[R]C=C1</smiles><smiles>C1CCNC1</smiles>

Nucleophile only<smiles>[R]/C=C/C=C/C=[N+]1CCCC1</smiles>

I
Not

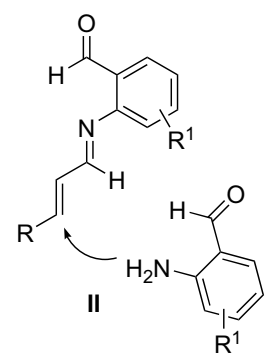

(2)

Scheme 2. 
Table 3. Direct organocatalytic domino aza-Michael/aldol condensation between 2-aminobenzaldehydes 1 and $\alpha, \beta$-unsaturated aldehydes $\mathbf{2}^{\mathrm{a}}$

$$
\text { (ldehyde }
$$

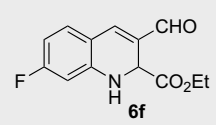

aExperimental conditions: A mixture of $1(0.3 \mathrm{mmol}), 2(0.25 \mathrm{mmol})$, benzoic acid (35 mol\%) and pyrrolidine (35 mol\%) in $1 \mathrm{ml}$ of DMSO was

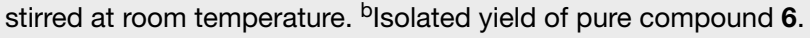

chloroform. Thus, we chose this reaction condition for the reaction between aldehyde $1 \mathrm{c}$ and different $\alpha, \beta$-unsaturated cyclic ketones 3 (Table 4).

The reactions gave the corresponding thioxanthenones derivatives 7 in 59-91\% yield. For example, the reaction between aldehyde 1c and 2-pentenone gave the corresponding thioxanthenone $7 \mathbf{c}$ in $82 \%$ yield (entry 3 ). The reaction can also be employed to gain access to thioxanthenones with a tertiary thio-center such as $\mathbf{7 d}$ (entry 4).

We next optimized the reaction between salicylic aldehyde 1a and 2-cyclohexenone 3b. After extensive screening, we found that we were able to furnish the corresponding tetrahydroxanthenone $\mathbf{8 a}$ in good yield by using pyrrolidine $(20 \mathrm{~mol} \%)$ as the catalyst and benzoic acid $(20 \mathrm{~mol} \%)$ as the additive in toluene. We next investigated the pyrrolidine-catalyzed reaction for a set of different 2-hydroxy-benzaldehyde derivatives 1 and $\alpha, \beta$-unsaturated cyclohexenones 3 (Table 5).

The organocatalytic domino reactions furnished the corresponding tetrahydroxanthenones $\mathbf{8 a - 8 g}$ in $59-85 \%$ yields. For example, the fluoro-substituted tetrahydroxanthenone $\mathbf{8 g}$ was synthesized in $78 \%$ yield (entry 7 ).

We propose the following mechanism for the pyrrolidine-catalyzed domino reac-
Table 4. Direct organocatalytic domino thio-Michael/aldol condensation between 2-mercaptobenzaldehyde 1 and $\alpha, \beta$-unsaturated ketones $\mathbf{3}^{\mathrm{a}}$

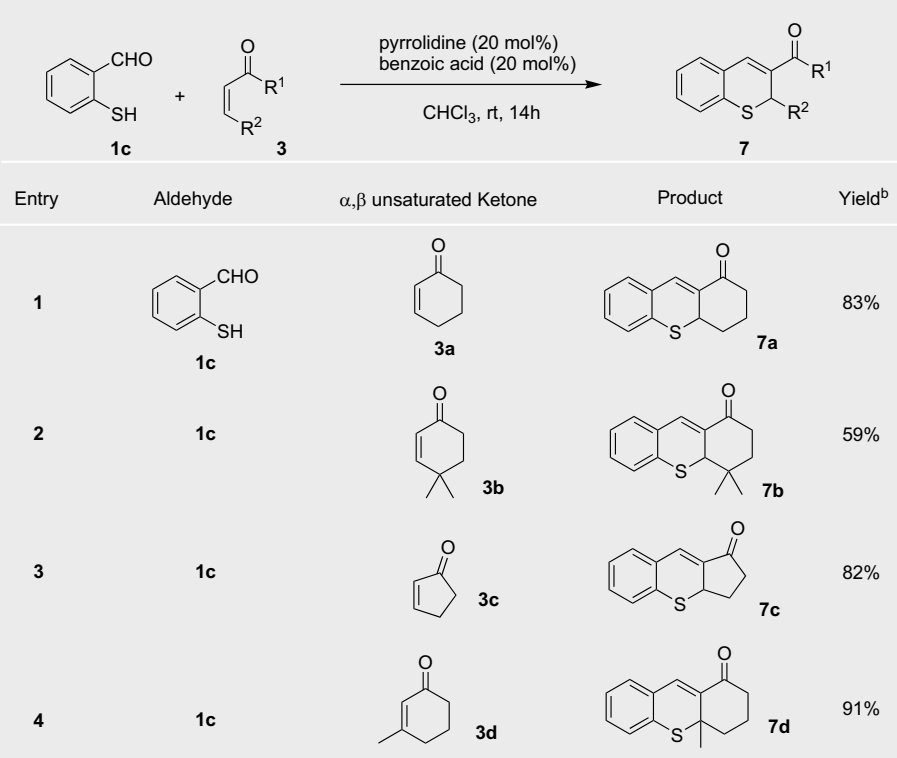

aExperimental conditions: A mixture of $1(0.3 \mathrm{mmol}), 3(0.25 \mathrm{mmol})$, benzoic acid $(20 \mathrm{~mol} \%)$ and pyrrolidine $(20 \mathrm{~mol} \%)$ in $1 \mathrm{ml} \mathrm{of} \mathrm{CHCl}_{3}$ was stirred at room temperature. lsolated yield of pure compound 7 .

Table 5. Direct organocatalytic domino oxo-Michael/aldol condensation between 2-hydroxybenzaldehydes 1 and $\alpha, \beta$-unsaturated ketones $\mathbf{3}^{\mathrm{a}}$

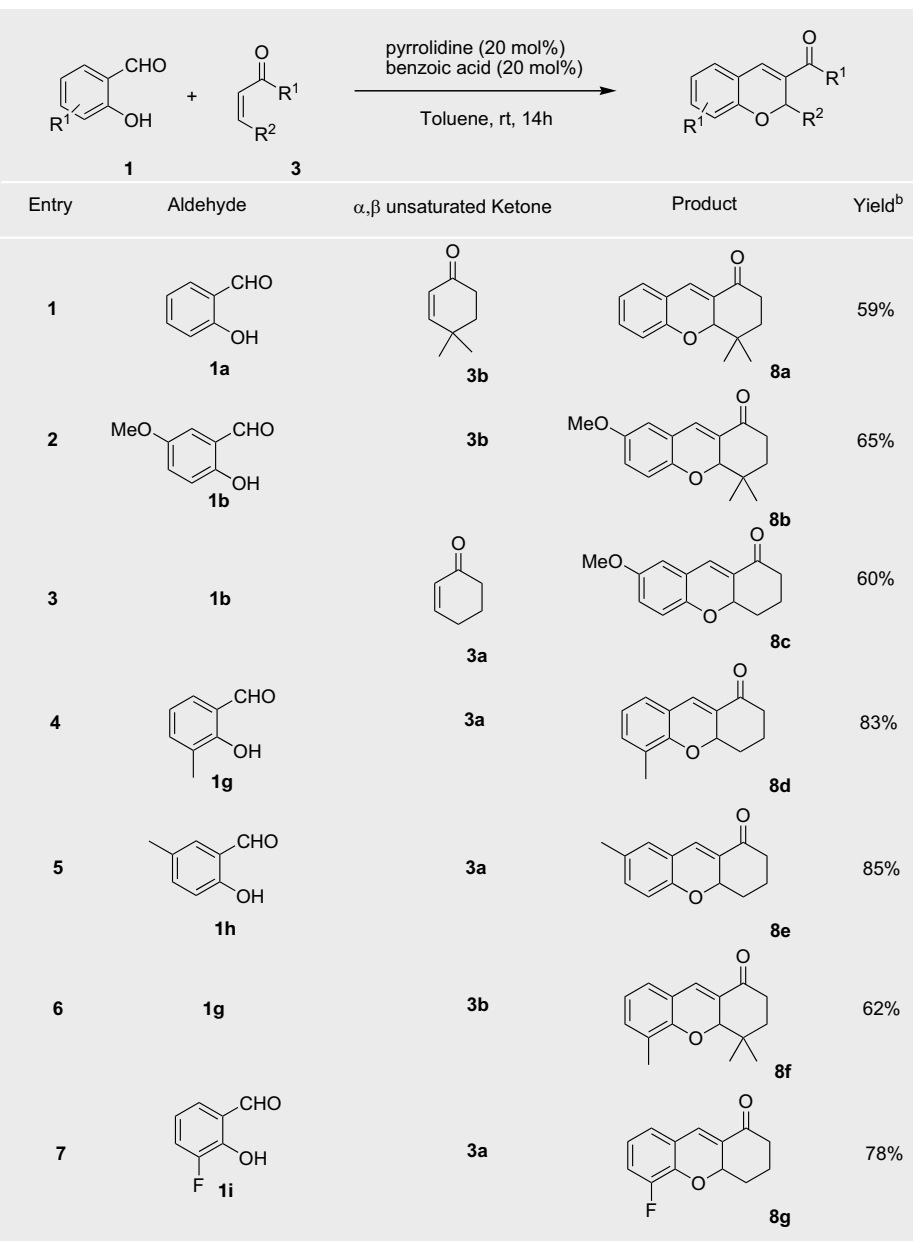

aExperimental conditions: A mixture of $\mathbf{1}(0.3 \mathrm{mmol}), \mathbf{3}(0.25 \mathrm{mmol})$, benzoic acid $(20 \mathrm{~mol} \%)$ and pyrrolidine $(20 \mathrm{~mol} \%)$ in $1 \mathrm{ml}$ of $\mathrm{CHCl}_{3}$ was stirred at room temperature. blsolated yield of pure compound $\mathbf{8}$. 
tions. The direct organocatalytic domino hetero-Michael/aldol condensation reaction starts with iminium activation of the $\alpha, \beta$-unsaturated aldehyde or ketone by the pyrrolidine. Next, a nucleophilic conjugate attack on the $\beta$-carbon of the enal or enone by the heteroatom of the benzaldehyde 1 results in an enamine intermediate (Scheme 3 ). The in situ generated enamine intermediate will next perform an intramolecular six-exo trig aldol addition, followed by hydrolysis of the resulting iminium intermediate to give the aldol product. Next, elimination of water gives the corresponding hetrocyclic product. This is further supported by the fact that the aldol intermediate was observed when the reaction was monitored by NMR analyses of the crude reaction mixture. The reactivity of the reaction is in accordance with the nucleophilicity of the in coming hetero-Michael nucleophile i.e. thiol $>$ aniline $>$ phenol.

In summary, we report a simple onepot organocatalytic domino hetero-Michael/aldol reaction. Pyrrolidine catalyzed the domino reactions between 2-hydroxy-, 2-mercapto- or 2-aminobenzaldehyde derivatives and $\alpha, \beta$-unsaturated aldehydes or ketones with high chemoselectivity and furnished the corresponding heterocyclic derivatives in good to high yields. Further application of this methodology in total synthesis and mechanistic studies are ongoing in our laboratory. ${ }^{[10]}$

\section{Experimental}

Typical experimental procedure for the reaction between 2-hetero substituted benzaldehydes and $\alpha, \beta$-unsaturated aldehydes: Pyrrolidine (0.0875 mmol, 0.35 equiv.), benzoic acid $(0.0875 \mathrm{mmol}, 0.35$ equiv.) and $1 \mathrm{ml}$ of DMSO were added to a round bottom flask. Next, the 2-heterosubstituted benzaldehyde 1 ( $0.3 \mathrm{mmol}, 1.2$ equiv.) and $\alpha, \beta$-unsaturated aldehyde $2(0.25 \mathrm{mmol}, 1$ equiv.) were added sequentially. The reaction was stirred at room temperature for 3 h. Next the crude reaction mixture was purified by column chromatography (Pentane: AcOEt mixtures) to afford the final compound.

Typical experimental procedure for the reaction between 2-mercaptobenzaldehyde $1 \mathrm{c}$ and $\alpha, \beta$-unsaturated ketones 3 : In a round bottom flask, pyrrolidine $(0.05 \mathrm{mmol}, 0.20$ equiv.), benzoic acid ( $0.05 \mathrm{mmol}, 0.20$ equiv.) were added in $1 \mathrm{ml}$ of $\mathrm{CHCl}_{3}$. Then 2-mercaptobenzaldehyde $(0.3 \mathrm{mmol}, 1.2$ equiv.) $1 \mathrm{c}$ and $\alpha, \beta$-unsaturated ketone 3 ( 0.25 mmol, 1 equiv.) were added sequentially. The reaction was stirred at room temperature over $14 \mathrm{~h}$. Next the crude reaction mixture was purified by column chromatography (pentane:AcOEt mixtures) to afford the final compound.

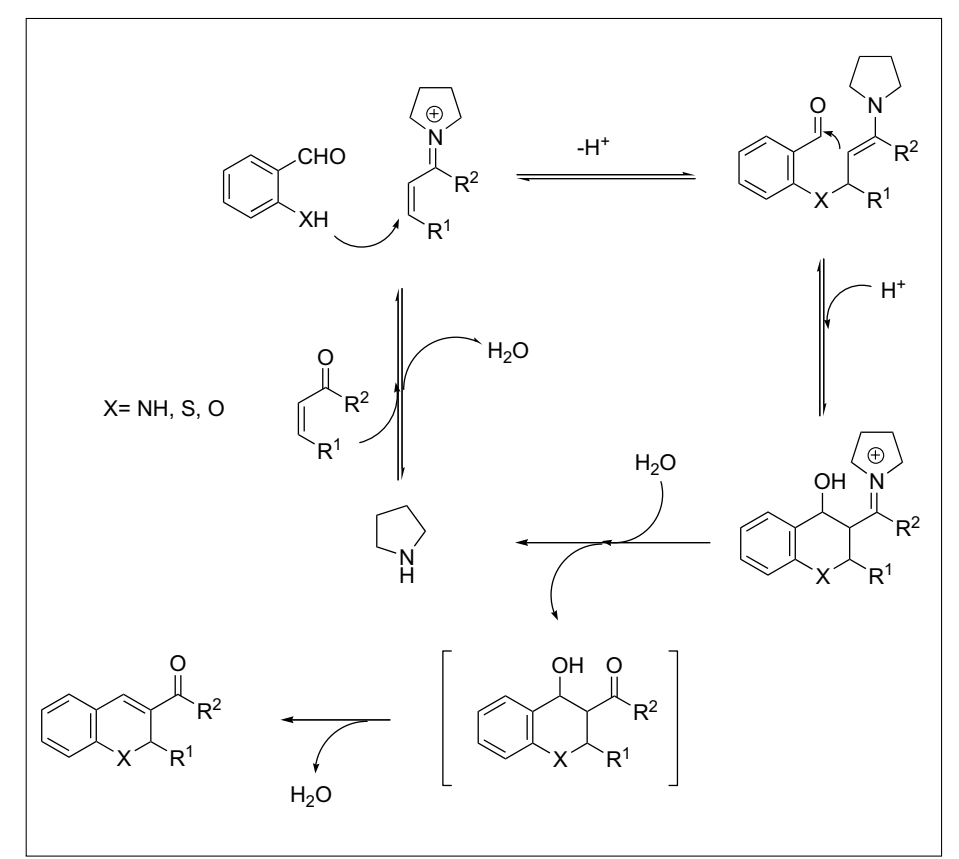

Scheme 3. A plausible reaction pathway for the organocatalytic heteroMichael/aldol reaction

Typical experimental procedure for the reaction between 2-hydroxybenzaldehydes derivatives $\mathbf{1}$ and $\alpha, \beta$-unsaturated ketones $\mathbf{3}$ : To a round bottom flask, pyrrolidine $(0.05$ mmol, 0.20 equiv.), benzoic acid (0.05 mmol, 0.20 equiv.) and $1 \mathrm{ml}$ of toluene were added sequentially. Next, the 2-hydroxy benzaldehyde derivative $(0.3 \mathrm{mmol}$, 1.2 equiv.) 1 and $\alpha, \beta$-unsaturated ketone 3 ( $0.25 \mathrm{mmol}, 1$ equiv.) were added sequentially. The reaction was stirred at room temperature for two days. Next the crude reaction mixture was purified by column chromatography (pentane:AcOEt mixtures) to afford the final compound.

The analytical data of some representative products are given below:

4d: ${ }^{1} \mathrm{H}$ NMR (300 MHz, $\left.\mathrm{CDCl}_{3}\right): \delta=1.18$ $(\mathrm{t}, J=6.9 \mathrm{~Hz}, 3 \mathrm{H}), 4.12(\mathrm{dq}, J=6.9,2.1$ $\mathrm{Hz}, 2 \mathrm{H}), 5.81(\mathrm{~s}, 1 \mathrm{H}), 6.96-7.04(\mathrm{~m}, 2 \mathrm{H})$, 7.24 (dd, $J=7.2,1.8 \mathrm{~Hz}, 1 \mathrm{H}), 7.32-7.37$ $(\mathrm{m}, 2 \mathrm{H}), 9.63(\mathrm{~s}, 1 \mathrm{H}) .{ }^{13} \mathrm{C}$ NMR $(75$ $\left.\mathrm{MHz}, \mathrm{CDCl}_{3}\right): \delta=14.0,61.9,70.5$, 117.1, 119.7, 122.6, 129.8, 130.0, 133.9, $140.8,155.0,168.7,188.9$. MALDITOF MS: 255.0634; $\mathrm{C}_{13} \mathrm{H}_{12} \mathrm{O}_{4}\left(\mathrm{M}+\mathrm{Na}^{+}\right.$: calcd 255.0633).

5a: ${ }^{1} \mathrm{H} \mathrm{NMR}\left(400 \mathrm{MHz}, \mathrm{CDCl}_{3}\right): \delta=9.66(\mathrm{~s}$, $1 \mathrm{H}), 7.46(\mathrm{~s}, 1 \mathrm{H}), 7.386(\mathrm{~d}, 1 \mathrm{H}, J=7 \mathrm{~Hz})$, 7.27-7.16 (m, 8H), $5.22(\mathrm{~s}, 1 \mathrm{H}) .{ }^{13} \mathrm{C}$ NMR $\left(100 \mathrm{MHz}, \mathrm{CDCl}_{3}\right): \delta=191.07$, $145.05,141.62,134.37,134.04,131.70$, $130.95,129.83,128.59,127.77,127.54$, 126.44, 125.80, 38.29. HRMS (ESI): calcd. for $\left(\mathrm{C}_{16} \mathrm{H}_{12} \mathrm{OS}+\mathrm{H}\right)=253.0682$. found 253.0677 .

6a: ${ }^{1} \mathrm{H}$ NMR (300 MHz, $\left.\mathrm{CDCl}_{3}\right): \delta=4.54$ $(\mathrm{s}, 1 \mathrm{H}), 5.68(\mathrm{~d}, J=1.9 \mathrm{~Hz}, 1 \mathrm{H}), 6.47(\mathrm{~d}$, $J=7.9,0.8 \mathrm{~Hz}, 1 \mathrm{H}), 6.66(\mathrm{dt}, J=7.9,0.8$ $\mathrm{Hz}, 1 \mathrm{H}), 7.12-7.18(\mathrm{~m}, 2 \mathrm{H}), 7.22-7.30$ (m, 4H), 7.36-7.41 (m, 2H), $9.49(\mathrm{~s}, 1 \mathrm{H})$.
${ }^{13} \mathrm{C}$ NMR $\left(75 \mathrm{MHz}, \mathrm{CDCl}_{3}\right): \delta=53.6$, 113.4, 117.4, 117.8, 126.1, 128.0, 128.7, $130.1,133.38,133.40,143.2,144.3$, 145.6, 190.4 .

7a: ${ }^{1} \mathrm{H} \mathrm{NMR}\left(400 \mathrm{MHz}, \mathrm{CDCl}_{3}\right): \delta=7.52(\mathrm{~d}$, $J=2.1 \mathrm{~Hz}, 1 \mathrm{H}), 7.40-7.10(\mathrm{~m}, 3 \mathrm{H}), 4.27$ (ddd, $J=2.4 \mathrm{~Hz}, J^{\prime}=5.7 \mathrm{~Hz}, J^{\prime \prime}=11.1 \mathrm{~Hz}$, $1 \mathrm{H}), 2.70-2.60(\mathrm{~m}, 1 \mathrm{H}), 2.50-2.40(\mathrm{~m}$, 1H), $2.30-1.80(\mathrm{~m}, 5 \mathrm{H}) .{ }^{13} \mathrm{C}$ NMR (100 $\left.\mathrm{MHz}, \mathrm{CDCl}_{3}\right): \delta=197.2,135.5,135.4$, $131.7,131.4,130.5,130.1,126.6,126.0$, 39.2, 38.3, 28.4, 20.9.

8c: ${ }^{1} \mathrm{HNMR}\left(400 \mathrm{MHz}, \mathrm{CDCl}_{3}\right): \delta=7.39(\mathrm{~d}$, $J=2.4 \mathrm{~Hz}, 1 \mathrm{H}), 6.82(\mathrm{~d}, J=1.6 \mathrm{~Hz}, 1 \mathrm{H})$, $6.74(\mathrm{t}, J=2.0 \mathrm{~Hz}, 1 \mathrm{H}), 4.92(\mathrm{~m}, 1 \mathrm{H}), 3.76$ $(\mathrm{s}, 3 \mathrm{H}), 2.62-2.35(\mathrm{~m}, 2 \mathrm{H}), 2.12-1.94(\mathrm{~m}$, 2H), $1.76-1.62(\mathrm{~m}, 2 \mathrm{H}) .{ }^{13} \mathrm{C}$ NMR (100 $\left.\mathrm{MHz}, \mathrm{CDCl}_{3}\right): \delta=197.7,154.7,150.2$, 131.8, 131.3, 122.8, 118.4, 116.9, 113.7, 74.8, 56.0, 39.1, 29.8, 18.2. HRMS(ESI): calcd. for $[\mathrm{M}+\mathrm{Na}]^{+}\left(\mathrm{C}_{14} \mathrm{H}_{14} \mathrm{O}_{3}\right)$ requires $\mathrm{m} / \mathrm{z} 253.0835$, found 253.0823 .

\section{Acknowledgements}

We gratefully acknowledge the Swedish National Research Council and Carl-Trygger Foundation for financial support.

Received: March 23, 2007

[1] a) E. A. Couladourous, A. T. Strongilos, Angew. Chem., Int. Ed. 2002, 41, 3677; b) Z. Gan, P. T. Reddy, S. Quevillon, S. Couve-Bonnaire, P. Arya, Angew. Chem., Int. Ed. 2005, 44, 1366; c) 'Comprehensive Heterocyclic Chemistry', Eds. A. R. Katrizky, C. W. Rees, Pergamon, Oxford, 1984, Vol.3, pp 737-883.

[2] a) V. K. Dua, V. P. Ojha, R. Roy, B. C. Joshi, N. Valecha, C. U. Devi, M. C. Bhatnagar, V. P. Sharma, S. K. Subbarao, J. 
Ethnopharmacol. 2004, 95, 247; b) H. Statzger, B. Schmidt, C. Root, W. Zinth, B. Fierz, F. Krieger, T. Kiefhaber, P. Gilch, J. Phys. Vhem. A 2004, 108, 10072; c) M. Itoigowa, C. Ito, H. Tokuda, F. Enjo, H. Nishinno, H. Furukawa, Cancer Lett. 2004, 214, 165; d) M. Goez, B. H. M. Hussein, PhysChemChemPhys 2004, 6, 5490; e) S. Milseni, M. Fagnoni, A. Albini, J. Org. Chem. 2005, 70, 603; f) K. C. Nicolau, J. A. Pferfferkorn, A. J. Roecker, G. Q. Cao, S. Barluenga, H. J. Mitchell, J. Am. Chem. Soc. 2000, 122, 9939; g) K. C. Nicolau, J. A. Pferfferkorn, S. Barluenga, H. J. Mitchell, A. J. Roecker, G. Q. Cao, J. Am. Chem. Soc. 2000, 122, 9968.

[3] a) J. S. Carter, B. Devadas, J. J. Talley, D. L. Brown, M. J. Graneto, D. J. Rogier Jr., S. R. Nagarajan, C. E. Hanau, S. J. Hartmann, C. L. Ludwid, S. Metz, D. E. Korte, S. R. Bertenshaw, M. G. Obukowicz, PCT Int. Appl. 2000, WO 2000023433 A1; b) J. S. Carter, M. G. Obukowicz, B. Devadas, J. J. Talley, D. L. Brown, M. J. Graneto, S. R. Bertenshaw, D. J. Rogier Jr., S. R. Nagarajan, C. E. Hanau, S. J. Hartmann, C. L. Ludwig, S. Metz, PCT Int. Appl. 1998, WO 9847890 A1; c) B. P. Chekal, E. Freund, C. M. Grill, E. Huthmann, M. Juza, V. Leshchinskaya, M. T. Maloney, L. M. Miller Jr., Y. Wang, M. Zeng, US Patent Appl. A1 20060020022, January 26th 2006; d) J. F. Geissler, J. L. Roesel, T. Meyer, U. P. Trinks, P. Traxler, N. B. Lydon, Cancer Res. 1992, 52, 4492; e) A. Bargagna, M. Longobardi, E. Mariani, P. Schenone, C. Losasso, G. Esposito, C. Falzarano, E. Marmo, Farmaco 1990, 45, 405; f) A. Arnoldi, A. Bonsignori, P. Melloni, L. Merlini, M. L. Quadri, A. C. Rossi, M. Valsecchi, J. Med. Chem. 1990, 33, 2865.

[4] a) S. W. Elmore, M. J. Coghlan, D. D. Anderson, J. K. Pratt, B. E. Green, A. X. Wang, M. A. Staschko, C. W. Lin, C. M. Tyree, J. N. Miner, P. B. Jacobson, D. M. Wilcox, B. C. Lane, J. Med. Chem. 2001, 44, 4481; b) P. D. Leeson, T. W. Carling, K. W. Moore, A. M. Moseley, J. D. Smith, G. Stevenson, T. Chan, R. Baker, A. C. Foster, S. Grimwood, J. A. Kemp, G. R. Marschall, K. Hoogsteen, J. Med. Chem. 1992, 35, 1954.

[5] For selected examples of recent methods for synthesis of chromenes see: a) I. Yavari, A. Ramazani, Synth. Commun. 1997, 27, 1385; b) S. Chang, R. H. Grubbs, $J$. Org. Chem. 1998, 63, 864; c) S. W. Youn, J. I. Eom, Org. Lett. 2005, 7, 3355; d) G. Lu, H. C. Malinakova, J. Org. Chem. 2004, 69, 4701; e) F. Kakiuchi, M. Yamauchi, N. Chantani, S. Murai, Chem Lett 1996, 25, 111; f) R. K. Thalji, K. A. Ahrendt, R. G. Bergman, J. A. Ellman, J. Am. Chem. Soc. 2001, 123, 9692; g) P. S. Bararn, E. J. Corey, J. Am. Chem. Soc. 2002, 124, 7904. Via arene metallation-Heck type reaction: h) N. Chatani, H. Inoue, T. Ikeda,
S. Murai, J. Org. Chem. 2000, 65, 4913. Via multiple bond activation-electrophilic substitution: i) U. Koch-Pomeranz, H. J. Hansen, H. Schmid, Helv. Chim. Acta 1973, 56, 2981; j) B. M. Trost, F. D. Toste, J. Am. Chem. Soc. 1996, 118, 6305; k) R. C. Larock, M. J. Dotty, Q. Tian, J. M. Zenner, J. Org. Chem. 1997, 62, 7536. By cyclization of aryl propargylic ethers: 1) J. Barluenga, M. Trincado, M. Marco-Arias, A. Ballesteros, E. Rubio, J. M. Gonzalez, Chem. Commun. 2005, 2008; m) S. A. Worlikar, T. Kersharwani, T. Yao, R. C. Larock, J. Org. Chem. 2007, 72, 1347. For selected examples of recent methods for synthesis of benzothiochromenes: $n$ ) J.-F. Bonfanti, D. Craig, Tetrahedron Lett. 2005, 46, 3719; o) P. T. Kaye, S. Afr. J. Sci. 2004, 100, 545; p) P. T. Kaye, X. W. Nocanda, Synthesis 2001, 2389; q) A. Arnoldi, M. Carughi, Synthesis 1998, 155; r) K. Kobayashi, H. Konishi, T. Kitamura, O. Morikawa, R. Nakahashi, J. Chem. Soc., Perkin Trans. 1 1999, 1547; s) G. V. Nenajdenko, A. V. Sanin, A. V. Churakov, J. A. K. Howard, E. S. G. Balenkova, Heterocycl. Compd. 1999, 35, 549. For selected examples of recent methods for synthesis of dihydroquinolidines: t) M. Takamura, K. Funabashi, M. Kanai, M. Shibasaki, J. Am. Chem. Soc. 2000, 122, 6327; u) M. Takamura, K. Funabashi, M. Kanai, M. Shibasaki, J. Am. Chem. Soc. 2001, 123, 6801; v) K. Makino, O. Hara, Y. Takiguchi, T. Katano, Y. Asakawa, K. Hatano, Y. Hamada, Tetrahedron Lett. 2003, 44, 8925; w) O. Hara, K. Sugimoti, K. Makino, Y. Hamada, Synlett 2004, 1625.

[6] a) B. Lesch, S. Bräse, Angew. Chem., Int. Ed. 2004, 43, 115; b) C. F. Nising, U. K. Ohnemüller, A. Friedrich, B. Lesch, J. Steiner, H. Schnöckel, M. Nieger, S. Bräse, Chem. Eur. J. 2006, 12, 3647; c) B. Lesch, S. Vanderheyden, S. Bräse, $A d v$. Synth. Catal. 2005, 347, 555; d) U. K. Ohnemüller, C. F. Nising, M. Nieger, S. Bräse, Eur. J. Org. Chem. 2006, 1535. For other examples see: a) P. T. Kaye, M. A. Musa, X. W. Nocada, R. S. Robinson, Org. Biom. Chem. 2003, 1, 1133; b) P. T. Kaye, S. Afr. J. Sciences 2004, 100, 545; c) P. T. Kaye, M. A. Musa, Synthesis 2002, 2701; d) Y. Satoh, J. L. Stanton, A. J. Hutchison, A. H. Libby, T. J. Kowalski, W. H. Lee, D. H. White, E. F. Kimble, J. Med. Chem. 1993, 36, 3580; e) G. -L. Zhao, Y. -L. Shi, M. Dhi, Org. Lett. 2005, 7, 4527 and references therein; f) G. -L. Zhao, J. -W. Huang, M. Shi, Org. Lett. 2003, 5, 4737.

[7] Y.-L. Shi, M. Shi, Synlett 2005, 2623.

[8] a) N. Halland, P. S. Aburell, K. A. Jørgensen, Angew. Chem., Int. Ed. 2004, 43, 1272; b) Y. Huang, A. M. Walji, C. H. Larsen, D. W. C. MacMillan, J. Am. Chem. Soc. 2005, 127, 15051; c) J. W. Yang, M. T. Hechavarria Fonseca, B. List, J. Am. Chem. Soc. 2005, 127, 15036; d) M. Marigo, T. Schulte, J. Franén, K. A. Jørgensen,
J. Am. Chem. Soc. 2005, 127, 15710; e) Y. Yamamoto, N. Momiyama, H. Yamamoto, J. Am. Chem. Soc. 2004, 126, 5962; f) D. B. Ramachary, N. S. Chowdari, C. F. Barbas III, Angew. Chem., Int. Ed. 2003, 42, 4233; g) H. Sundén, I. Ibrahem, L. Eriksson, A. Córdova, Angew. Chem., Int. Ed. 2005, 44, 4877; h) D. Enders, M. R. M. Hüttl, C. Grondal, G. Raabe, Nature 2006, 441,861 .

[9] For an excellent review on domino reactions see: L. F. Tietze, Chem. Rev. 1996, 96, 115.

[10] Inspired by this research, we and others have developed asymmetric methods for the synthesis of heterocycles based on the concept presented in this paper: a) For asymmetric chromene synthesis see: a) $\mathrm{H}$. Sundén, I. Ibrahem, G.-L. Zhao, L. Eriksson, A. Córdova, Chem. Eur. J. 2007, 13, 574. Other examples in the literature: b) T. Govender, L. Hojabri, F. M. Moghaddam, P. I. Arvidsson, Tetrahedron Asymm. 2006, 17, 1763; c) H. Li, J. Wang, T. E-Nunu, L. Zu, W. Jiang, S. Wei, W. Wang, Chem. Commun. 2007, 507. For asymmetric thiochromene synthesis see: d) R. Rios, H. Sundén, I. Ibrahem, G.-L. Zhao, L. Eriksson, A. Córdova, Tetrahedron Lett. 2006, 47, 8547; e) W. Wang, H. Li, J. Wang, L. $\mathrm{Zu}$, J. Am. Chem. Soc. 2006, 128, 10354. For 1,2-dihydroquinolidine synthesis see: f) H. Sundén, R. Rios, I. Ibrahem, G.-L. Zhao, L. Eriksson, A. Córdova, Adv. Synth. Cat. 2007, 349, 827; g) H. Li, J. Wang, H. Xie, L. Zou, W. Jiang, E. N. Duesler, W. Wang, Org. Lett. 2006, in press. For tetrahydrothioxanthenone synthesis see: h) R. Rios, H. Sundén, I. Ibrahem, G.L. Zhao, A. Córdova, Tetrahedron Lett. 2006, 47, 8679. For tetrahydroxanthenone synthesis see: i) R. Rios, H. Sundén, I. Ibrahem, G.-L. Zhao, A. Córdova, Tetrahedron Lett. 2007, 48, 2181. 